\title{
Designing Adaptive Argumentation Learning Systems Based on Artificial Intelligence
}

\author{
THIEMO WAMBSGANSS
}

University of St.Gallen, St.Gallen, Switzerland

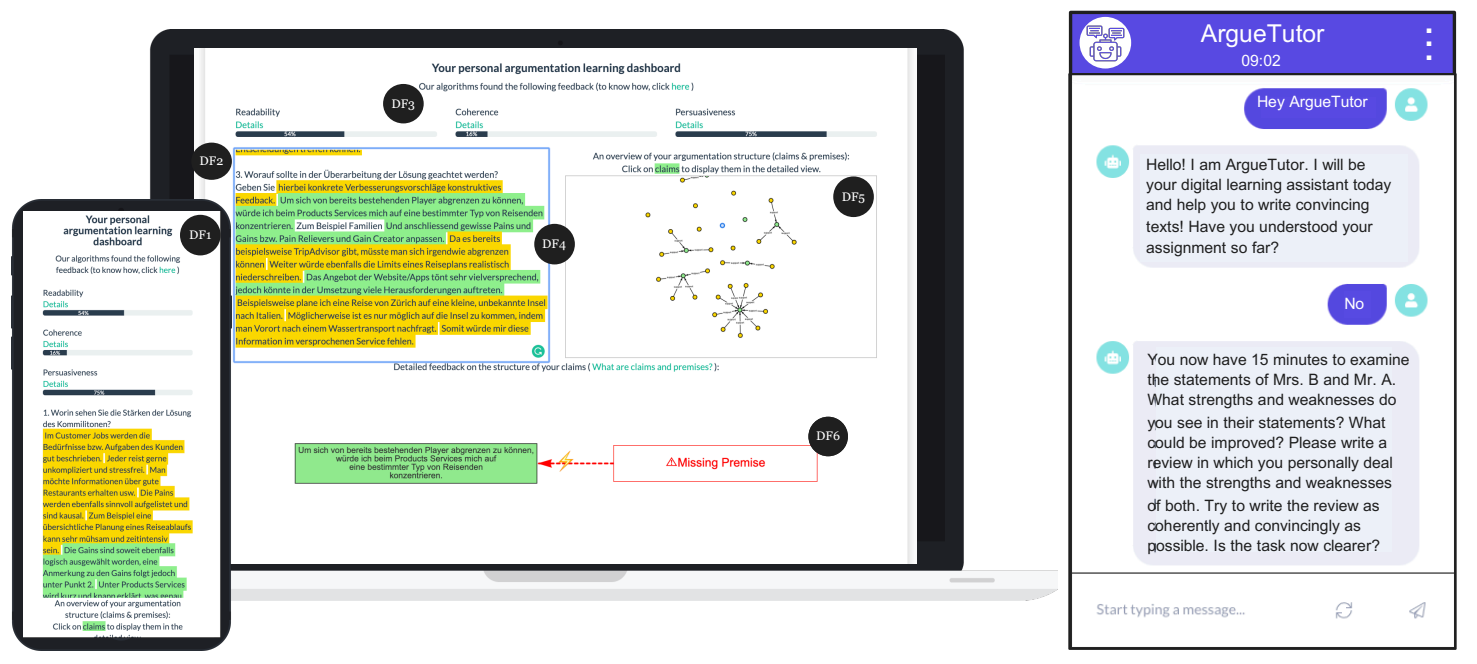

Figure 1: Left: A user is receiving adaptive argumentation writing support by our system $A L$ while conducting a peer-review exercise in a large-scale lecture scenario. Right: A user is receiving adaptive argumentation tutoring with intelligent feedback and theory input by our conversational learning tutor ArgueTutor.

\begin{abstract}
:
Argumentation skills are an omnipresent foundation of our daily communication and thinking. However, the learning of argumentation skills is limited due to the lack of individual learning conditions for students. Within this dissertation, I aim to explore the potential of adaptive argumentation skill learning based on Artificial Intelligence (AI) by designing, implementing, and evaluating new technology-enhanced pedagogical concepts to actively support students in developing the ability to argue in a structured, logical, and reflective way. I develop new student-centered pedagogical scenarios with empirically evaluated design principles, linguistic corpora, ML algorithms, and innovative learning tools based on an adaptive writing support system and a pedagogical conversational agent. My results indicate that adaptive learning tools based on ML algorithms and user-centered design patterns help students to develop better argumentation writing skills. Thereby, I contribute to research by bridging the boundaries of argumentation learning and argumentation mining and by examining pedagogical scenarios for adaptive argumentation learning from a user-centered perspective.
\end{abstract}

Keywords and Phrases: Argumentation Learning, Adaptive Skill Learning, Argumentation Mining, Pedagogical Conversational Agents 


\section{CONTEXT AND MOTIVATION}

In today's world, most information is readily available, and solely reproducing information is losing attention. This results in a shift of job profiles towards interdisciplinary, ambiguous, and creative tasks [3]. Educational institutions need to evolve in their curricula when it comes to the composition of skills and knowledge conveyed [35]. Especially teaching higher-order thinking skills to students, such as critical thinking, collaboration, or problem-solving, has gained in importance [8]. This has also been recognized by the Organization for Economic Co-operation and Development (OECD), which included these skills as a major element of their Learning Framework 2030 [21]. One subclass represents the skill of arguing in a structured, reflective, and well-formed way [36]. Argumentation is not only an essential part of our daily communication and thinking but also contributes significantly to the competencies of communication, collaboration, and problem-solving [18]. Starting with studies from Aristotle, the ability to form convincing arguments is recognized as the foundation for persuading an audience of novel ideas and plays a major role in strategic decision-making and analyzing different standpoints, especially in regard to managing digitally enabled organizations. To develop skills such as argumentation, it is of great importance for the individual student to receive continuous feedback throughout their learning journey, also called formative feedback $[2,13]$. Thus, institutions, such as universities, are facing the challenge of providing individual learning conditions, since every student would need a personal tutor to have an optimal learning environment to learn how to argue [38]. However, this is unsurprisingly hindered due to traditional large-scale lectures or due to the growing field of distance learning scenarios such as massive open online courses (MOOCs, [29]). One possible solution to this dilemma is using adaptive argumentation learning tools that provide individual feedback on the argumentation of students' texts, e.g., through adaptive writing support systems or pedagogical conversational agents. These intelligent tutoring systems (ITS) often imitate meaningful, individual instructor-learner interactions (e.g., [16]) and have been successfully used in adaptively supporting learners to conduct a task by mimicking the gold standard of human tutors (e.g., $[43,46,49])$. By using such systems, students would be able to learn autonomously and independently of the instructor, time, and place $[33,42]$.

More specifically, a promising way to support students to learn structured argumentation and enable teachers to convey it to classes of large sizes and independent from location might be the usage of adaptive technologybased applications in a pedagogical scenario for a student's learning journey. Therefore, recent advances in Artificial Intelligence (AI) such as argumentation mining (AM) seem to be a promising approach, since Natural Language Processing (NLP) and Machine Learning (ML) offer a scalable analysis approach to identify and classify the argumentation in texts, e.g., to access individual levels of argumentation [39,41]. This has been applied to various other types of texts, such as court statements [20], newspaper articles [4,7] or user-generated content [12]. Researchers, especially from the fields of educational technology, have designed tools to support the active teaching of argumentation to students with input masks or representational guidelines to enhance students' learning of argumentation (e.g., $[11,22,24])$. However, literature falls short of providing an approach with principles and proof on how to design and embed an adaptive argumentation learning tool in a pedagogical scenario to help students learn how to argue with intelligent formative feedback provided by an algorithm based on NLP and ML. 


\section{KEY RELATED WORK}

Researchers have designed and evaluated several tools based on input masks and representational guidelines to support the active writing process of high school students. This has been investigated across a variety of fields, including law [24], science [22,34], and conversational argumentation [11]. However, literature related to research on adaptive argumentation learning is scarce [42]. Adaptive support approaches for argumentation learning (e.g., $[17,24,30,32])$ describe a rather new field of argumentation learning supported by technologyenhanced systems. The aim is to provide pedagogical feedback to a learner's actions and solutions, hints, and recommendations to encourage and guide future activities in the writing processes or automated evaluation to indicate whether an argument is syntactically and semantically correct. However, the combination of NLP, ITS, and pedagogically evaluated formative feedback in a student's learner journey is hardly investigated, due to its fhigh complexity. As Scheuer (2015), p. 126 identifies, "rigorous empirical research with respect to adaptation strategies is almost absent; a broad and solid theoretical underpinning, or theory of adaptation for collaborative and argumentative learning is still lacking". There is a clear lack of design knowledge and empirical studies for pedagogical scenarios for adaptive argumentation learning (e.g., [28,33,42]). Besides, conversational agents (CA) offer new forms of providing individual guidance and feedback to students [27,50,51], for instance, when completing a task to write persuasive texts, through a natural conversation interface combined with AM technology. However, in "literature exists no approach with principles, design knowledge, and evaluation on how to design and embed an adaptive dialog-based argumentation tutoring system in a pedagogical scenario to help students to learn how to argue" $[16,43,48]$.

\section{RESEARCH QUESTIONS AND METHODOLOGY}

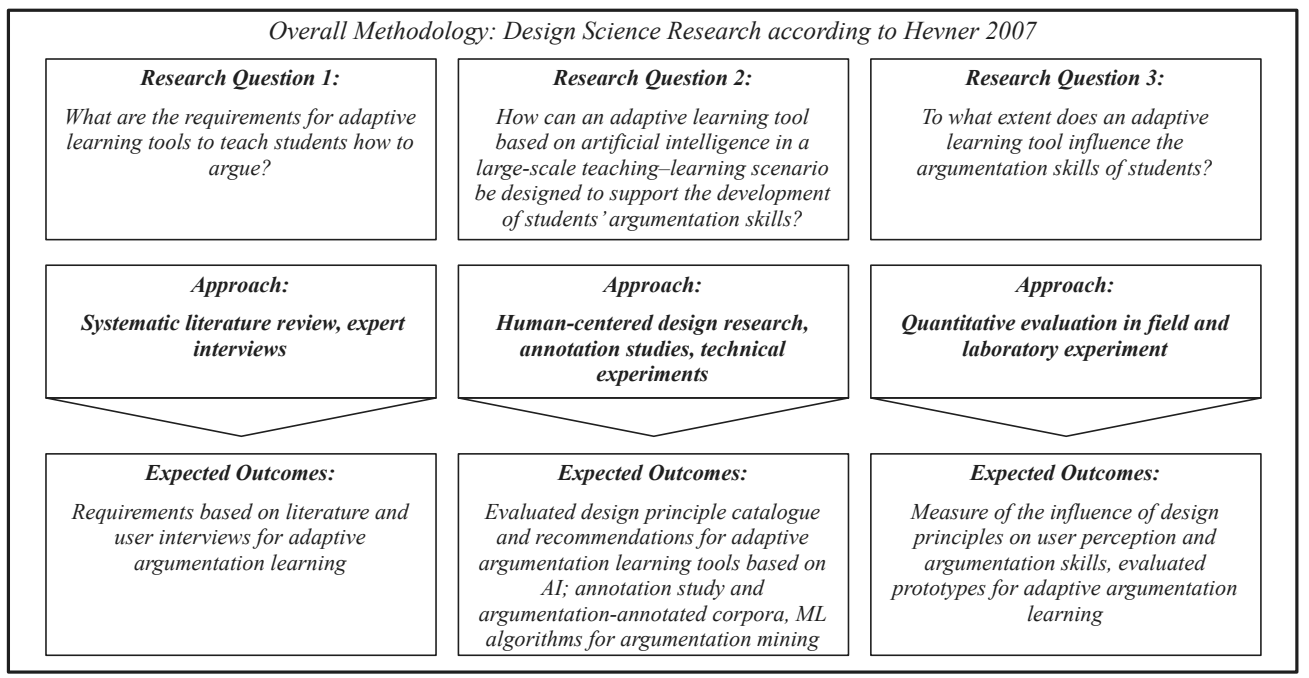

Figure 2: Research questions and methodology

This dissertation is guided by a design-oriented research (DSR) approach [14]. I decided to follow this approach in order to use a scientific method to solve a set of practical problems that researchers and practitioners experience in their own practice and to contribute to the existing body of knowledge by designing and evaluating new research artifacts. Based on the described research gaps in the related work section, I aim to contribute to adaptive argumentation learning by answering the following research questions (RQ) (illustrated in Figure 2): 
RQ1: What are the requirements for adaptive learning tools to teach students how to argue?

RQ2: How can an adaptive learning tool based on artificial intelligence in a large-scale teaching-learning scenario be designed to support the development of students' argumentation skills?

RQ3: To what extent does an adaptive learning tool influence the argumentation skills of students?

I intend to iteratively design and evaluate IT-learning artifacts on the baseline of existing theory (cognitive dissonance based on [9]) informing the artifact design [15]. I believe cognitive dissonance theory could explain why formative feedback on a student's argumentation will motivate the student to learn how to argue [9]. Dissonance can be an initial trigger for a student's learning process and thus the construing of new knowledge structures [23] through critical reflection, also reflected in the literature on transformation learning (e.g., [19]) or self-regulation theory [1]. However, the right portion of cognitive dissonance is very important for the motivation to solve it. According to Festinger, individuals might not be motivated enough to resolve it if the dissonance is too obvious, whereas a high level of dissonance might lead to frustration. Therefore, I believe that the right level of feedback to a student skill, such as argumentation skills, could lead to cognitive dissonance and thus to motivation to change the behavior, belief, or knowledge to learn how to argue.

\section{RESULTS AND CONTRIBUTION TO DATE}

Up to now, I was able to conduct several research steps that contribute to my three questions. For example, I investigated user and theory requirements for adaptive argumentation learning tools [42-44] for RQ1 and RQ2 and argumentation-annotated corpora [41], transfer learning algorithms for argumentation mining [39], and the effects of an adaptive argumentation learning tool on students' ability to write persuasive business model peer reviews $[40,43]$ for $R Q 3$. The preliminary results of my findings are embedded in two iteratively developed learning tools (see Figure 1). First, I developed AL (short for Argumentation Learning), an adaptive writing support system that helps students to improve their argumentation skills in peer-feedback scenarios [40]. Second, I developed ArgueTutor (short for Argumentation Tutoring), an adaptive dialog-based tutoring system that provides students with adaptive and instant feedback, theoretical input, and step-by-step guidance during their writing process [43]. I will provide a brief overview of preliminary results regarding the specific RQ1. However, I would encourage the interested reader to find further details in the corresponding papers (e.g., [3943]).

Preliminary results regarding RQ1: I followed two different development approaches: 1) a rigorous theorymotivated approach, where I systematically searched literature in the field of educational technology and $\mathrm{HCl}$ (following $[3,6,47])$ to carefully derive requirements and principles for the design of adaptive argumentation learning tools; and 2), a user-centred design approach, where I conducted a series of 42 semi-structured interviews (following $[5,10]$ ) with students from my university to derive user stories.

Preliminary results regarding RQ2: The results from RQ1 served as theory and user requirements for the design of adaptive argumentation learning systems. Hence, I derived different design principles for a) the design of an adaptive writing support system and $b$ ) the design of a dialog-based tutoring system, since both artifact types differ fundamentally in the user interaction (dialog-based vs. static interaction). Based on the initial findings, I tested the design principles as a design hypothesis according to the build-measure-learn paradigm of [25]. With several low-fidelity prototypes (e.g., [45]), I further learned about the interaction of the different 
learning systems and the way students want to learn and improve their argumentation skills (more information in [40]).

From a technical perspective on designing an adaptive argumentation learning tool, it is crucial to develop an algorithm that is able to provide individual and adaptive feedback based on the argumentation level of students. Since no suitable corpora were available, I created a new dataset for argumentation skill learning. Therefore, 1) I derived an annotation scheme for a new data domain for AM based on argumentation theory (and previous work on annotation schemes for persuasive student essays (e.g., [31])), 2) presented an annotation study based on 50 persuasive peer reviews and three annotators to show that the annotation of student peer reviews is reliably possible, and 3) created a final and freely available corpus of 1,000 student peer reviews collected in our lecture about business innovation in German [41]. The corpus now serves as a reliable database for the training of ML algorithms for the design of adaptive argumentation learning systems in German.

Preliminary results regarding RQ3: Based on my design principles, the tested prototypes, the corpus development, and trained models, I designed two instantiations of an adaptive argumentation learning tool in different pedagogical scenarios. First, I built $A L$, an adaptive learning support system for argumentation skills [41], and evaluated it against a proven argumentation tool in a laboratory experiment with 54 students [40]. I found that students using AL wrote more convincing texts with better formal quality of argumentation and better subjective persuasiveness compared to the ones using the traditional approach. Second, I built ArgueTutor, an adaptive dialog-based tutoring system that provides students with adaptive and instant feedback, theoretical input, and step-by-step guidance during their writing process [43]. I evaluated ArgueTutor in a laboratory experiment with 55 students and found that ArgueTutor helps students to write formally more convincing texts compared to a static argumentation learning tool (the design and evaluation of ArgueTutor is currently under review at $\mathrm{CHI} 2021)$.

\section{EXPECTED NEXT STEPS}

For future work, I aim to further refine $\mathrm{AL}$ and ArgueTutor to evaluate to what extent an adaptive argumentation writing support system and an adaptive argumentation tutoring system can influence students' argumentation skills (RQ3). Therefore, I plan to conduct two further studies (study 1 and study 2):

Study 1: I aim to compare AL and ArgueTutor in a laboratory experiment. My objective is to contribute to research with empirical results on the effects of a static writing support tool vs. a dialog-based tutoring tool on students' formal and informal argumentation skills as well as on effects on their perception. Therefore, I will conduct a laboratory experiment with three groups to evaluate the impact of $\mathrm{AL}$ and ArgueTutor on the development of students' argumentation quality. I will rely on one control group (participants will not receive any learning tool) and two treatment groups. Participants in treatment group 1 use AL for conducting a persuasive writing exercise, whereas participants in treatment group 2 will conduct the exact same exercise using ArgueTutor. I will carefully ensure that I only manipulate the interaction type (static vs. conversational). Both tools will be trained with the same feedback algorithm in the back end. Finally, I aim to measure the formal and informal argumentation level of the text from the participants and compare their perception of the learning process regarding motivation, cognitive dissonance, and technology acceptance using key constructs (e.g., [37]). 
Study 2: Next, I want to embed both tools in a large-scale lecture scenario to evaluate the long-term effect of an adaptive argumentation learning tool on students' meta-argumentation skills. This can be achieved with a longitudinal study in a real-world learning setting. Therefore, particularly for analyzing the long-term effect of using ArgueTutor and AL, I aim to implement both artifacts into our existing learning management system [26] and measure long-term effects on the usability and the acceptance of skill learning during the complete threemonth life cycle of a lecture. I want to investigate the hypothesis that adaptive argumentation learning tools influence the long-term meta-argumentation skills of students. At the end of the study, I want to contribute to research with two evaluated learning tools that can be used in a learning-teaching scenario where students do a certain writing exercise and receive adaptive argumentation writing support or tutoring in the writing process.

\section{CONCLUSION AND EXPECTED CONTRIBUTION}

In this dissertation project, I aim to design, build, and evaluate two new forms of adaptive argumentation learning tools ( $A L$ and ArgueTutor) that provide students with feedback on the argumentation structure of a text independent of an instructor, time, and place by leveraging the recent advances of AM algorithms.

Besides the software artifact as a situated implementation of an adaptive argumentation learning tool, I will contribute design knowledge to the scientific knowledge base. The resulting design knowledge is not only valid for my specific case but can also be transferred to further use cases in adaptive argumentation learning. For instance, it is easily possible to apply the concept of $A L$ in courses that deal with other content than business models or other languages. For this purpose, only the back-end algorithm needs to be adapted to the other scenario. Multiple corpora and AM models exist in literature that can be easily embedded in AL, e.g., for English student essays [33] or English law cases [20]. The design principles of form and function and the overall system design do not need to be adapted for those use cases. Furthermore, it is also possible to transfer the design knowledge to pedagogical scenarios that target the training of other metacognition skills. For instance, if the learning of general feedback skills or empathy skills of students is aimed to be trained, a similar adaptive writing support system can be used. However, in this case, the system design might need to be revised partially, e.g., the graph visualization or the argumentation dashboard need to be adapted.

Moreover, research in argumentation learning and AM has made great strides in the last decade. In both research streams, we now know a lot about the mechanisms, the potential benefits, and shortcomings. However, both research fields have not yet crossed their boundaries to provide a comprehensive and multidisciplinary connection between argumentation learning for students and AM technology. I bridge these boundaries by examining a pedagogical scenario for adaptive argumentation learning from both perspectives, human-computer interaction and computational linguistics. Therefore, I contribute not only new rich argumentation annotation schemes, an argumentation corpus, and novel ML models but also insights into the overall embedding and design of adaptive argumentation learning tools and the potential of dialog-based argumentation learning. These insights will provide a new perspective on Al-based adaptive argumentation and more general metacognition skill learning, which has been neglected so far in existing research.

All in all, my research offers design knowledge to further improve educational feedback applications based on intelligent algorithms. With further advances in NLP and ML, I hope my work will attract researchers to design more intelligent tutoring systems for other learning scenarios or metacognition skills and thus contribute to the OECD Learning framework 2030 towards a metacognition-skill-based education. 


\section{REFERENCES}

1. Albert Bandura. 1991. Social cognitive theory of self-regulation. Organizational Behavior and Human Decision Processes 50, 2 : 248-287. https://doi.org/10.1016/0749-5978(91)90022-L

2. Paul Black and Dylan Wiliam. 2009. Developing the theory of formative assessment. Educational Assessment, Evaluation and Accountability 21, 1: 5-31. https://doi.org/10.1007/s11092-008-9068-5

3. Jan vom Brocke, Alexander Simons, Kai Riemer, Bjoern Niehaves, Ralf Plattfaut, and Anne Cleven. 2015. Standing on the shoulders of giants: Challenges and recommendations of literature search in information systems research. Communications of the Association for Information Systems 37, 1: 205-224. https://doi.org/10.17705/1cais.03709

4. Glenn Rowe Chris Reed Raquel Mochales Palau and Marie-Francine Moens. 2008. Language Resources for Studying Argument. In Proceedings of the Sixth International Conference on Language Resources and Evaluation (LREC'08).

5. Mike Cohn. 2004. User Stories Applied For Agile Software Development.

6. Harris M. Cooper. 1988. Organizing knowledge syntheses: A taxonomy of literature reviews. Knowledge in Society 1, 1: $104-126$. https://doi.org/10.1007/BF03177550

7. Lingjia Deng and Janyce Wiebe. 2015. MPQA 3.0: An Entity/Event-Level Sentiment Corpus. In Proceedings of the 2015 Conference of the North American Chapter of the Association for Computational Linguistics: Human Language Technologies, 1323-1328.

8. Charles Fadel, Maya Bialik, and Bernie Trilling. 2015. Four-dimensional education : the competencies learners need to succeed.

9. Leon Festinger. 1962. Cognitive Dissonance. Scientific American 207, 4: 93-106. https://doi.org/10.1038/scientificamerican106293

10. Jochen. Gläser and Grit. Laudel. 2010. Experteninterviews und qualitative Inhaltsanalyse : als Instrumente rekonstruierender Untersuchungen. VS Verlag für Sozialwiss. Retrieved February 8, 2018 from http://www.springer.com/de/book/9783531172385

11. R De Groot, R Drachman, R Hever, B Schwartz, U Hoppe, A Harrer, M De Laat, R Wegerif, B M Mclaren, and B Baurens. 2007. Computer Supported Moderation of E-Discussions: the ARGUNAUT Approach. Retrieved January 11, 2019 from http://www.argunaut.org

12. Ivan Habernal and Iryna Gurevych. 2016. What makes a convincing argument? empirical analysis and detecting attributes of convincingness in web argumentation. In Proceedings of the 2016 conference on empirical methods in natural language processing, 1214-1223.

13. John Hattie and Helen Timperley. 2007. The Power of Feedback. Review of Educational Research 77, 1: 81-112. https://doi.org/10.3102/003465430298487

14. Alan R Hevner. 2007. A three cycle view of design science research. Scandinavian Journal of Information Systems: 1-6. Retrieved from http://aisel.aisnet.org/cgi/viewcontent.cgi?article=1017\&context=sjis

15. Alan R Hevner, Salvatore T March, Jinsoo Park, and Sudha Ram. 2004. Design Science in Information Systems Research. Design Science in IS Research MIS Quarterly 28, 1: 75. Retrieved April 11, 2019 from https://wise.vub.ac.be/sites/default/files/thesis_info/design_science.pdf

16. Sebastian Hobert and Raphael Meyer Von Wolff. 2019. Say Hello to Your New Automated Tutor - A Structured Literature Review on Pedagogical Conversational Agents. 14th International Conference on Wirtschaftsinformatik, Siegen, Germany.

17. Chenn Jung Huang, Shun Chih Chang, Heng Ming Chen, Jhe Hao Tseng, and Sheng Yuan Chien. 2016. A group intelligencebased asynchronous argumentation learning-assistance platform. Interactive Learning Environments 24, 7: 1408-1427. https://doi.org/10.1080/10494820.2015.1016533

18. Deanna Kuhn. 1992. Thinking as Argument. Harvard Educational Review 62, 2: 155-179. https://doi.org/10.17763/haer.62.2.9r424r0113t670l1

19. Jack Mezirow. 1991. Transformative dimensions of adult learning. Jossey-Bass, San Francisco, CA 94104-1310.

20. Raquel Mochales Palau and Aagje leven. 2009. Creating an argumentation corpus: do theories apply to real arguments? $\{\mathrm{A}\}$ case study on the legal argumentation of the $\{\mathrm{ECHR}$. In Proceedings of the Twelfth International Conference on Artificial Intelligence and Law (ICAIL 2009), Twelfth international conference on artificial intelligence and law (ICAIL 2009)., Barcelona, Spain, 8-12 June 2009, 21-30. 
OECD. 2018. The Future of Education and Skills - Education 2030. https://doi.org/2018-06-15

22. Jonathan F. Osborne, J. Bryan Henderson, Anna MacPherson, Evan Szu, Andrew Wild, and Shi Ying Yao. 2016. The development and validation of a learning progression for argumentation in science. Journal of Research in Science Teaching 53, 6: 821-846. https://doi.org/10.1002/tea.21316

23. Jean Piaget, Terrance Brown, and Kishore Julian Thampy. 1986. The Equilibration of Cognitive Structures: The Central Problem of Intellectual Development. Jean Piaget, Terrance Brown, Kishore Julian Thampy. American Journal of Education 94, 4: 574-577. https://doi.org/10.1086/443876

24. Niels Pinkwart, Kevin Ashley, Collin Lynch, and Vincent Aleven. 2009. Evaluating an Intelligent Tutoring System for Making Legal Arguments with Hypotheticals. IOS Press. Retrieved January 11, 2019 from http://iaiedsoc.org/pub/1302/file/19_4_05_Pinkwart.pdf Eric Ries. 2011. The Lean Startup: How Today's Entrepreneurs Use Continuous Innovation to Create Radically Successful Businesses. Crown Books.

26. Roman Rietsche, Kevin Duss, Jan Martin Persch, and Matthias Söllner. 2018. Design and Evaluation of an IT-based Formative Feedback Tool to Foster Student Performance Understanding and Designing Trust in Information Systems View project Future of Collaboration View project. In Thirty Ninth International Conference on Information Systems. Retrieved January 11, 2019 from https://www.researchgate.net/publication/329450233

27. Sherry Ruan, Liwei Jiang, Justin Xu, Bryce Joe-Kun Tham, Zhengneng Qiu, Yeshuang Zhu, Elizabeth L. Murnane, Emma Brunskill, and James A. Landay. 2019. QuizBot: A Dialogue-based Adaptive Learning System System for Factual Knowledge. Chi: 1-13. https://doi.org/10.1145/3290605.3300587

28. Oliver Scheuer. 2015. Towards adaptive argumentation learning systems. Retrieved January 11, 2019 from https://www.researchgate.net/publication/298087259

29. Julia E. Seaman, I. E. Allen, and Jeff Seaman. 2018. Higher Education Reports - Babson Survey Research Group. Retrieved February 17, 2020 from http://www.onlinelearningsurvey.com/highered.html

30. Christian Stab and Iryna Gurevych. 2014. Identifying Argumentative Discourse Structures in Persuasive Essays. In Conference on Empirical Methods in Natural Language Processing (EMNLP 2014)(Oct. 2014), Association for Computational Linguistics, p.(to appear), 46-56. Retrieved October 23, 2018 from www.ukp.tu-darmstadt.de

31. Christian Stab and Iryna Gurevych. 2014. Annotating Argument Components and Relations in Persuasive Essays. In Proceedings of COLING 2014, the 25th International Conference on Computational Linguistics: Technical Papers, 1501-1510. Retrieved October 17, 2018 from http://www.ukp.tu-darmstadt.de

32. Christian Stab and Iryna Gurevych. 2017. Recognizing Insufficiently Supported Arguments in Argumentative Essays. Retrieved October 23, 2018 from www.ukp.tu-darmstadt.de

33. Christian Stab and Iryna Gurevych. 2017. Parsing Argumentation Structures in Persuasive Essays. Computational Linguistics 43, 3: 619-659. https://doi.org/10.1162/COLI_a_00295

34. Daniel D Suthers and Christopher D Hundhausen. 2001. European Perspectives on Computer-Supported Collaborative Learning. Retrieved January 11, 2019 from http://lilt.ics.hawaii.edu/papers/2001/Suthers-Hundhausen-Euro-CSCL-2001.pdf

35. Heikki Topi. 2018. Using competencies for specifying outcome expectations for degree programs in computing: Lessons learned from other disciplines. 2018 SIGED International Conference on Information Systems Education and Research.

36. Stephen E. Toulmin. 2003. The uses of argument: Updated edition. https://doi.org/10.1017/CBO9780511840005

37. Viswanath Venkatesh and Hillol Bala. 2008. Technology acceptance model 3 and a research agenda on interventions. Decision Sciences 39, 2: 273-315. https://doi.org/10.1111/j.1540-5915.2008.00192.x

38. Lev Semenovich Vygotsky. 1980. Mind in society: The development of higher psychological processes. Harvard university press.

39. Thiemo Wambsganss, Nikolaos Molyndris, and Matthias Söllner. 2020. Unlocking Transfer Learning in Argumentation Mining: A Domain-Independent Modelling Approach. In 15th International Conference on Wirtschaftsinformatik. https://doi.org/10.30844/wi_2020_c9-wambsganss

40. Thiemo Wambsganss, Christina Niklaus, Matthias Cetto, Matthias Söllner, Jan Marco Leimeister, and Siegfried Handschuh. 2020. AL : An Adaptive Learning Support System for Argumentation Skills. In ACM CHI Conference on Human Factors in Computing Systems, 1-14. 
41. Thiemo Wambsganss, Christina Niklaus, Matthias Söllner, Siegfried Handschuh, and Jan Marco Leimeister. 2020. A Corpus for Argumentative Writing Support in German. In 28th International Conference on Computational Linguistics (Coling).

42. Thiemo Wambsganss and Roman Rietsche. 2020. Towards designing an adaptive argumentation learning tool. In 40th International Conference on Information Systems, ICIS 2019, 1-9.

43. Thiemo Wambsganss, Matthias Söllner, and Jan Marco Leimeister. 2020. Design and Evaluation of an Adaptive Dialog-Based Tutoring System for Argumentation Skills. In International Conference on Information Systems (ICIS).

44. Thiemo Wambsganss, Florian Weber, Tobias Staufenberg, Leopold Bott, and Matthias Söllner. 2020. Systematische Entwicklung eines Lerntools zur Erhöhung der Argumentationsfähigkeiten von Studierenden. In 15th International Conference on Wirtschaftsinformatik, 449-464. https://doi.org/10.30844/wi_2020_d6-wambsganss

45. Thiemo Wambsganss, Rainer Winkler, Pascale Schmid, and Matthias Söllner. 2020. Unleashing the Potential of Conversational Agents for Course Evaluations: Empirical Insights from a Comparison with Web Surveys. In Twenty-Eighth European Conference on Information Systems (ECIS2020), 1-18.

46. Thiemo Wambsganss, Rainer Winkler, Matthias Söllner, and Jan Marco Leimeister. 2020. A Conversational Agent to Improve Response Quality in Course Evaluations. In ACM CHI Conference on Human Factors in Computing Systems.

47. Jane Webster and Richard T Watson. 2002. Analyzing the past to prepare for the future: Writing a literature review Reproduced with permission of the copyright owner . Further reproduction prohibited without permission . MIS Quarterly 26, 2: xiii-xxiii. https://doi.org/10.1.1.104.6570

48. R. Winkler and M. Söllner. 2018. Unleashing the Potential of Chatbots in Education : A State-Of-The-Art Analysis . In : Academy of Management. Meeting, Annual Chicago, A O M. Retrieved from https://www.alexandria.unisg.ch/254848/1/JML_699.pdf

49. Rainer Winkler, Claudio Büchi, and Matthias Söllner. 2019. Improving Problem-Solving Skills with Smart Personal Assistants: Insights from a Quasi Field Experiment. Fortieth International Conference on Information Systems (ICIS), Oecd 2014: 1-17.

50. Rainer Winkler, Sebastian Hobert, Antti Salovaara, Matthias Söllner, and Jan Marco Leimeister. 2020. Sara, the Lecturer: Improving Learning in Online Education with a Scaffolding-Based Conversational Agent. In Conference on Human Factors in Computing Systems - Proceedings. https://doi.org/10.1145/3313831.3376781

51. N. Zierau, T Wambsganss, Andreas Janson, Sofia Schöbel, and Jan Marco Leimeister. 2020. The Anatomy of User Experience with Conversational Agents : A Taxonomy and Propositions of Service Clues. In: International Conference on Information Systems (ICIS). 